\title{
A novel method for downstream characterization of breast cancer circulating tumor cells following CellSearch isolation
}

Henrik Frithiof ${ }^{1}$, Charlotte Welinder ${ }^{1}$, Anna-Maria Larsson ${ }^{1,2}$, Lisa Rydén ${ }^{3,4^{*}+}$ and Kristina Aaltonen ${ }^{1+}$

\begin{abstract}
Background: Enumeration of circulating tumor cells (CTCS) obtained from minimally invasive blood samples has been well established as a valuable monitoring tool in metastatic and early breast cancer, as well as in several other cancer types. The gold standard technology for detecting CTCs in blood against a backdrop of millions of leukocytes is the FDA-approved CellSearch system (Janssen Diagnostics), which relies on EpCAM-based immunomagnetic separation. Secondary characterization of these cells could enable treatment selection based on specific targets in these cells, as well as providing a real time window into the metastatic process and offering unique insights into tumor heterogeneity. The objective of this study was to develop a method for downstream characterization of CTCs following isolation with the CellSearch system.

Methods: An in vitro CTC model system focusing on clinically useful treatment predictive biomarkers in breast cancer, specifically the estrogen receptor a (ERa) and the human epidermal growth factor receptor 2 (HER2), was established using healthy donor blood spiked with breast cancer cell lines MCF7 (ERa $\left.{ }^{+} / \mathrm{HER2}{ }^{-}\right)$and SKBr3 $\left(\mathrm{ERa}^{-} / \mathrm{HER2}^{+}\right)$. Following CTC isolation by CellSearch, the captured CTCS were further enriched and fixed on a microscope slide using the in-house-developed CTC-DropMount technique.
\end{abstract}

Results: The recovery rate of CTCs after CellSearch Profile analysis and CTC-DropMount was $87 \%$. A selective and consistent triple-immunostaining protocol was optimized. Cells positive for DAPI, cytokeratin (CK) 8, 18 and 19, but negative for the leukocyte-specific marker CD45, were classified as CTCs and subsequently analyzed for ERa and HER2 expression. The method was verified in breast cancer patient samples, thus demonstrating its clinical relevance.

Conclusions: Our results show that it is possible to ascertain the status of important predictive biomarkers expressed in breast cancer CTCs using the newly developed CTC-DropMount technique. Downstream characterization of multiple biomarkers using a standard fluorescence microscope demonstrates that important clinical and biological information may be obtained from a single patient blood sample following either CellSearch epithelial or profile analyses.

Trial registration: Clinical Trials NCT01322893

Keywords: Circulating tumor cells (CTC), Metastatic breast cancer, CellSearch, Biomarkers, Estrogen receptor (ER), Human epidermal growth factor 2 (HER2), Immunofluorescence

\footnotetext{
* Correspondence: lisa.ryden@med.lu.se

${ }^{\dagger}$ Equal contributors

${ }^{3}$ Division of Surgery, Department of Clinical Sciences, Lund University, Lund, Sweden

${ }^{4}$ Department of Surgery, Skåne University Hospital, Lund, Sweden

Full list of author information is available at the end of the article
} 


\section{Background}

During the last decade, enumeration of circulating tumor cells (CTCs) in peripheral blood was established as a prognostic tool for predicting time-to-recurrence and survival in metastatic and early breast cancer, as well as in several other cancer types [1-4]. The gold standard technology, and the only platform implemented on a larger scale, is the FDA-approved semi-automated CellSearch technology (Jansen Diagnostics, Raritan, NJ, USA). In CellSearch, enriched EpCAM-positive CTCs are defined as nucleated cells positive for cytokeratin (CK) 8, 18 and 19 , but negative for the leukocyte-specific surface protein, CD45. However, the field of CTC research is now moving beyond solely quantifying cells in peripheral blood. Phenotypic and molecular characterization of CTCs has the potential to provide clinically important information from an easily accessible blood sample, a 'liquid biopsy'. Serial blood sampling followed by molecular characterization can provide insights into tumor progression and enable early detection of treatment resistance.

In breast cancer, assessment of estrogen receptor $\alpha$ $(E R \alpha)$ status in the primary tumor is crucial in classification and treatment prediction [5]. Determining receptor status identifies patients eligible for endocrine therapy, which remains the mainstay adjuvant treatment for $E R \alpha^{+}$breast cancers, either as monotherapy or in conjunction with chemotherapy. Although an $\mathrm{ER}^{+}$primary tumor is a common trait and found in approximately $80 \%$ of patients with primary breast cancer, it is no guarantee for a favorable outcome following endocrine treatment as recurrence rates of $19-41 \%$ are observed at 10 years following 5 years of tamoxifen [6-8]. Moreover, in metastatic breast cancer, approximately $40-50 \%$ of patients fail to respond to endocrine treatment, despite an initially positive assay [9]. The causes of this considerable inconsistency are multifactorial and have not been entirely elucidated, but discordance in ER $\alpha$ status between the primary tumor and involved lymph nodes or distant metastases has been established in $6-30 \%$ of studied cases and may contribute to treatment resistance [10-14]. In fact, this phenotypical shift is associated with significantly shorter median survival for patients with metastatic disease when compared with consistent ER $\alpha$ positivity in disease progression [10]. Additionally, it has been reported that the majority of CTCs in patients with $\mathrm{ER}^{+}$primary tumors are in fact $\mathrm{ER \alpha ^{- }}$ prior to therapy, with a concordance of less than 30\% [15-17].

Human epidermal growth factor receptor 2 (HER2) is a tyrosine kinase receptor encoded by a proto-oncogene located on chromosome 17 (17q12), and is the second most important predictive biomarker in breast cancer $[18,19]$. Amplification of this gene occurs in approximately $10-30 \%$ of primary breast cancers, correlating with poor prognosis and an aggressive phenotype $[18,19]$. This subgroup of patients benefits from immunotherapy with an HER2targeted monoclonal antibody, trastuzumab, in combination with chemotherapy in adjuvant, neoadjuvant and metastatic settings $[20,21]$. Similar to the dynamic progression observed in $\mathrm{ER}^{+}$tumors, the HER2 status of metastases can differ from that of the primary tumor [22]. Discordance has been observed in $7-14 \%$ of studied cases [11,23-25]. It has been shown that patients with HER2 ${ }^{-}$ tumors might acquire HER2 amplification during disease progression, as demonstrated by isolation of $\mathrm{HER} 2^{+}$CTCs in patients with an HER2 ${ }^{-}$primary tumor [26-29]. Another explanation for the discrepancy in biomarker expression between primary tumors and CTCs may be tumor heterogeneity. Tumor clones shed into the blood stream are more likely to represent those with most malignancy, exemplified by HER2-amplified clones, despite the primary tumor being diagnosed as HER2 normal. These patients are less likely to receive HER2-targeted treatment, although a complete or partial response has been observed in selected cases [30]. Two prospective trials including patients with HER2-negative primary tumors and HER2positive CTCs are currently open for recruitment and aim to elucidate whether trastuzumab will have a beneficial effect on these cases [31].

Thus, treatment decisions based on the phenotype of the primary tumor alone might omit critical facts relevant to the prognosis and choice of treatment. Biopsies from metastatic sites are not always available for practical reasons and are inevitably accompanied by an invasive procedure. CTCs are easily accessible from a normal blood sample, and since CTCs are shed from multiple metastatic sites as well as from the primary tumor, characterization of these cells could provide important information for treatment prediction.

The aim of this study was to establish a method for downstream characterization of multiple treatment predictive markers expressed by CTCs after CellSearch-based selection, without the necessity of additional patient samples. Validation of the method in samples from patients with metastatic breast cancer highlights the potential of the clinical utility of this technique.

\section{Methods}

\section{In vitro model}

Breast cancer cell lines MCF7 and SKBr3 were obtained from the American Type Culture Collection (ATCC/LGC Standards GmbH, Wesel, Germany) and were used to establish an in vitro model system for CTC characterization following CellSearch isolation. MCF7 expresses ER $\alpha$ but is negative for HER2 amplification. Contrary, SKBr3 cells are HER2-positive and negative for ER $\alpha$. MCF7 cells were grown in a $5.0 \% \mathrm{CO}_{2}$ incubator under UV-light at $37^{\circ} \mathrm{C}$ in culture vessels containing $5 \mathrm{~mL}$ MEM/EBSS (HyClone Laboratories, Inc., Utah, United States) medium 
supplemented with $1 \%$ sodium pyruvate, $1 \%$ non-essential amino acids, $10 \%$ fetal bovine serum (FBS) and $1 \%$ penicillin streptomycin mixture (Pen-Strep) for MCF7, and RPMI 1640 (HyClone Laboratories, Inc.), while SKBr3 cells were cultured under the same conditions in $5 \mathrm{~mL}$ MEM/EBSS plus 10\% FBS and 1\% Pen-Strep. Harvesting of cells was performed at approximately $80-90 \%$ confluency after 5-10 min trypsinization.

Healthy donor blood samples were processed within $24 \mathrm{~h}$ from withdrawal, and spiking of cells occurred in conjunction with subsequent CellSearch analyses. Two different spiking methods were used. First, dilution of cells resulted in approximately 2000 cells per $7.5 \mathrm{ml}$ blood, and using the CTC-DropMount technique (described below), approximately 200 cells were applied to 10 individual slides, which were later used in the optimization of staining procedures. Second, to ascertain the recovery rate of the method, a specific number of cells were harvested individually with a $10 \mu \mathrm{L}$ pipette under a bright-field microscope equipped with a standard achromatic $\times 10 / 0.25$ objective. In detail, a fraction of the cell culture was transferred to a Petri dish containing cell culture medium. While observing the cell culture suspension through the eyepieces of the microscope, suitable individual cells were selected and carefully extracted using a $10 \mu \mathrm{L}$ pipette before transfer to a healthy donor blood sample. Since the process is continuously monitored by microscopy, one can confirm that the cell has been properly extracted. Reference values of 5,15 , and 50 cells were selected. Independently collected duplicates of each of the three respective cell quantities were added to $7.5 \mathrm{~mL}$ of healthy donor blood samples and processed according to the specified method. The agreement between the measured results and the reference values was calculated to define the recovery rate.

\section{Fixation of samples using CTC-DropMount}

CellSearch Profile (Jansen Diagnostics) analysis was performed according to the manufacturer's protocol, which involves enrichment of CTCs with magnetic ferrofluidassociated anti-EpCAM antibodies but no consecutive staining. The enriched samples were mounted on slides using a specific procedure developed in-house, CTCDropMount. The solution containing isolated CTCs (approximately $900 \mu \mathrm{L}$ ) was transferred to an $1.5 \mathrm{~mL}$ Eppendorf tube and placed in a magnetic tray. After $10 \mathrm{~min}$ incubation, the non-adherent solvent was extracted. The cells were resuspended in $10 \mu \mathrm{L} 1 \times \mathrm{PBS}$, mounted on superfrost slides (ThermoScientific, Germany) and incubated at $37^{\circ} \mathrm{C}$ for $30 \mathrm{~min}$. Fixation was accomplished by immersing slides in pure methanol for $5 \mathrm{~min}$. The samples were stored at $-20^{\circ} \mathrm{C}$.

The CTC-DropMount method was also used for enriched cells after standard CellSearch epithelial cell analysis (i.e. all cells were semi-automatically stained with CK-phycoerythrin (PE), CD45-allophycocyanin (APC) and DAPI in a procedure described previously (3)). In this case, the solution containing enriched CTCs was extracted from the CellSearch cartridge after complete analyses, and the cartridge was carefully rinsed with $1 \times$ PBS buffer to ensure maximum extraction before transfer to a $1.5 \mathrm{~mL}$ Eppendorf tube in a magnetic tray. An overview of the CTC-DropMount method is provided in Figure 1.

\section{Immunostaining protocol for ERa and HER2}

Following cell permeabilization using Dako Target Retrieval solution containing Tris/EDTA buffer solution pH 9.0 and detergent (S2368, Dako Denmark A/S, Glostrup, Denmark), slides were stained according to the optimized protocols detailed in Tables 1 and 2, respectively. Briefly, to define CTCs against the backdrop of remaining leukocytes, a CD45-specific AlexaFluor647-labeled mouse monoclonal antibody (F10894, AbD Serotec, Oxford, UK) was assessed at dilutions of 1:1, 1:5 and 1:10 [32]. The primary antibody used against $E R \alpha$ was a rabbit monoclonal antibody (RM-9101-S1, Thermo Scientific, Fremont, CA, USA), diluted 1:50 [33]. Secondary detection was accomplished with AlexaFluor488-labeled goat anti-rabbit antibody (A-11034, Life Technologies, Carlsbad, CA, USA) at dilutions of 1:200, 1:300, 1:400, and 1:500. For detection of cytokeratin, slides were incubated with CellSearch Staining Reagent containing a PE-labeled mouse monoclonal antibody specific to CK 8,18 and 19 , at a concentration of $0.0006 \%$ (Janssen Diagnostics). Antibody dilutions were made with DAKO Antibody Diluent containing 1\% FBS in PBS and 0.1\% detergent (S2022, Dako). Slides were finally mounted with coverslips and counterstained with an antifade reagent containing the nucleic acid dye, DAPI, (S36942, Life Technologies), thus enhancing resistance to photobleaching.

The level of HER2-expression was investigated using a primary monoclonal rabbit antibody specific for the human HER2 oncoprotein (1:250; EP1045Y, Abcam plc, Cambridge, UK), along with a polyclonal porcine antirabbit secondary antibody conjugated with alkaline phosphatase (ALP) (1:50; D0306, Dako). Detection was accomplished based on the ALP-Fast Red reaction, using the substrate and chromogen in Liquid Permanent Red (LPR) (K0640, Dako [34]). Incubation times assessed for the ALP-Fast Red reaction were 5, 10 and, $15 \mathrm{~min}$. LPR was evaluated by both fluorescence and bright-field microscopy.

Immunofluorescence and bright-field analyses were performed with an Olympus BX63 microscope equipped with a dual color/monochrome digital DP80 camera (Olympus Optical CO., Hamburg, Germany). Single pass filters for DAPI, GFP/Alexa488, PE/TxRed and APC/ 


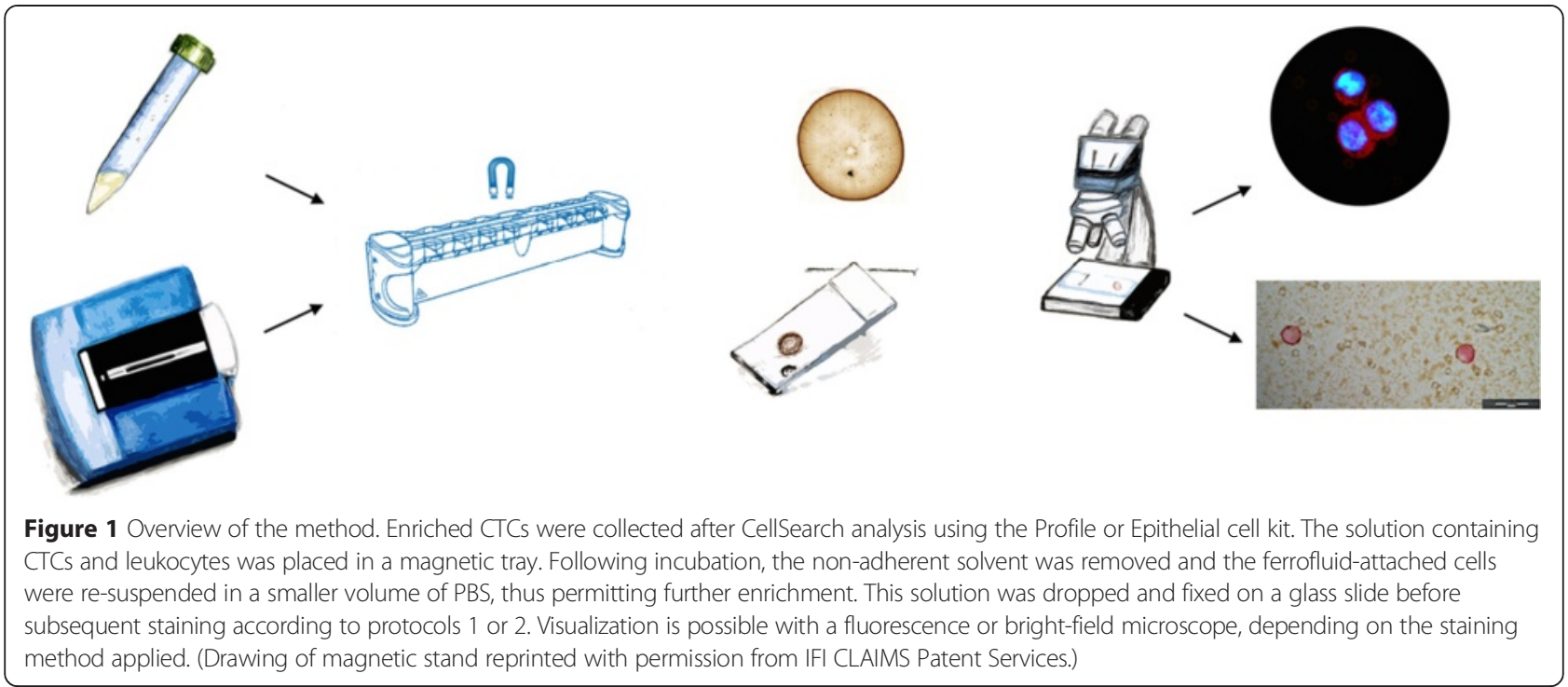

Alexa647/Cy5 were used for staining evaluation. In a few cases, an Olympus BX51 microscope (Olympus Optical CO) and a Zeiss Axio Observer Z1 microscope (Carl Zeiss Microscopy, LLC., NY, USA) outfitted with single pass filters for each individual fluorochrome were used for examination.

\section{In vivo validation}

Patient blood samples were investigated for clinical validation of the technique. All patients had metastatic breast cancer and had been included in the ongoing CTC-MBC trial (Clinical Trial Id. NCT01322893) at Lund University, Sweden. In total, nine clinical samples of $7.5 \mathrm{~mL}$ whole blood from nine individual patients were assessed by CellSearch profile analysis. CTC-DropMount and subsequent staining according to the staining protocols detailed in Tables 1 and 2 were performed prior to evaluation by fluorescence and bright-field microscopy. All patient sample analyses were processed in conjunction with positive and negative controls, decreasing the risk of methodological errors, as well as confirming successful staining reactions. Ethical permission for the CTC-MBC study was obtained from Lund University Ethical Board (EPN 2010/135) and all patients gave written informed consent.

Table 1 Optimized staining protocol for ERa, CK, and CD45 expression in CTCs and leukocytes

\begin{tabular}{|c|c|c|c|c|}
\hline Step & Reagent & Concentration & Interval & Manufacturer/Batch \\
\hline 1. Cell fixation & Methanol & $1: 1$ & $\begin{array}{l}5 \text { min } \\
\text { at RT }\end{array}$ & $\begin{array}{l}\text { Merck KGaA, Germany, } \\
\# 1659409\end{array}$ \\
\hline 2. Cell permeabilization & $\begin{array}{l}\text { Dako Envision Target Retrieval solution }{ }^{\mathrm{TM}} \text { (50x) (Tris/EDTA } \\
\text { buffer solution, } \mathrm{pH} \text { 9.0, and detergent) }\end{array}$ & $1: 50$ & $\begin{array}{l}20 \mathrm{~min} \\
\text { at } 37^{\circ} \mathrm{C}\end{array}$ & $\begin{array}{l}\text { Dako Denmark, A/S, } \\
\text { \#20000821 }\end{array}$ \\
\hline 3. $A B$ serum & Dako AB diluent ${ }^{\mathrm{TM}}$ (1\% FBS in PBS, $0.1 \%$ detergent) & $1: 1$ & $\begin{array}{l}20 \mathrm{~min} \\
\text { at RT }\end{array}$ & $\begin{array}{l}\text { Dako Denmark, A/S, } \\
\# 00091216\end{array}$ \\
\hline $4^{1}$. ERa labelling & Rabbit monoclonal AB specific to ERa & $1: 50$ & $\begin{array}{l}60 \mathrm{~min} \\
\text { at } 37^{\circ} \mathrm{C}\end{array}$ & $\begin{array}{l}\text { Thermo Scientific, United } \\
\text { States, \#9101S1210D }\end{array}$ \\
\hline $5^{1}$. CD45 staining & $\begin{array}{l}\text { Alexa Fluor } 647 \text { labeled mouse monoclonal AB specific to } \\
\text { CD45 }\end{array}$ & $1: 5$ & $\begin{array}{l}60 \mathrm{~min} \\
\text { at } 37^{\circ} \mathrm{C}\end{array}$ & AbD Serotec, UK, \#B173123 \\
\hline $6^{2}$. ERa staining & Alexa Fluor 488 labeled goat anti-rabbit secondary AB & $1: 200$ & $\begin{array}{l}45 \mathrm{~min} \\
\text { at } \mathrm{RT}\end{array}$ & $\begin{array}{l}\text { Life Technologies, United } \\
\text { States, \#1423009 }\end{array}$ \\
\hline $\begin{array}{l}7^{2} . \text { Cytokeratin } 8,18 \\
\text { and } 19 \text { staining }\end{array}$ & $\begin{array}{l}\text { Phycoerythrin labeled mouse monoclonal AB, specific to CK } 8 \text {, } \\
18 \text {, and } 19\end{array}$ & $0.0006 \%$ & $\begin{array}{l}45 \mathrm{~min} \\
\text { at RT }\end{array}$ & $\begin{array}{l}\text { Janssen Diagnostics, United } \\
\text { States, \#E491A }\end{array}$ \\
\hline $\begin{array}{l}\text { 8. Nuclear } \\
\text { counterstaining }\end{array}$ & Slowfade ${ }^{\oplus}$ Gold antifade with nuclear dye, DAPI & & & $\begin{array}{l}\text { Life Technologies, United } \\
\text { States, \#1500156 }\end{array}$ \\
\hline
\end{tabular}

Washing with iced PBS $10.0 \%$, v/v, $3 \times 3$ min between each step.

Abbreviations: AB - antibody, DAPI - 4',6-diamidino-2-phenylindole dihydrochloride, EDTA - Ethylenediaminetetraacetic acid, ERa - estrogen receptora,

PBS - phosphate buffered saline, RT - room temperature, Tris - 2-Amino-2-hydroxymethyl-propane-1,3-diol.

${ }^{1}$ Step 4 and 5 can be performed simultaneously.

${ }^{2}$ Step 6 and 7 can be performed simultaneously. 
Table 2 Optimized staining protocol for HER2 expression

\begin{tabular}{|c|c|c|c|c|}
\hline Step & Reagent & Concentration & Interval & Manufacturer/Batch \\
\hline 1. Cell fixation & Methanol & 1:1 & $\begin{array}{l}5 \mathrm{~min} \\
\text { at RT }\end{array}$ & Merck KGaA, Germany, \#1659409 \\
\hline $\begin{array}{l}\text { 2. Cell } \\
\text { permeabilization }\end{array}$ & $\begin{array}{l}\text { Dako Envision Target Retrieval solution }{ }^{\mathrm{TM}} \text { (50X) (Tris/EDTA } \\
\text { buffer solution, pH 9.0, and detergent) }\end{array}$ & $1: 50$ & $\begin{array}{l}20 \min \\
\text { at } 37^{\circ} \mathrm{C}\end{array}$ & Dako Denmark, A/S, \#20000821 \\
\hline 3. $A B$ serum & Dako AB diluent ${ }^{\mathrm{TM}}$ (1\% FBS in PBS, $0.1 \%$ detergent) & $1: 1$ & $\begin{array}{l}20 \mathrm{~min} \\
\text { at RT }\end{array}$ & Dako Denmark, A/S, \#00091216 \\
\hline 4. HER2 labelling & Rabbit monoclonal AB specific to HER2 & 1:250 & $\begin{array}{l}20 \min \\
\text { at RT }\end{array}$ & $\begin{array}{l}\text { Abcam plc, United Kingdom, } \\
\text { \#GR122507-5 }\end{array}$ \\
\hline 5. ALP conjugation & ALP-conjugated porcine polyclonal anti-rabbit $A B$ & 1:50 & $\begin{array}{l}30 \min \\
\text { at RT }\end{array}$ & Dako Denmark, A/S, \#20008362 \\
\hline 6. LPR & Red chromogen and substrate buffert & $\begin{array}{l}\text { 1:100 } \\
\text { (chromogen:substrate) }\end{array}$ & $\begin{array}{l}10 \mathrm{~min} \\
\text { at RT }\end{array}$ & Dako Denmark, A/S, \#10082175 \\
\hline $\begin{array}{l}\text { 7. Nuclear } \\
\text { counterstaining }\end{array}$ & Slowfade ${ }^{\circledast}$ Gold antifade with nuclear dye, DAPI & & & $\begin{array}{l}\text { Life Technologies, United States, } \\
\# 1500156\end{array}$ \\
\hline
\end{tabular}

Washing with iced PBS $10.0 \%(\mathrm{v} / \mathrm{v}), 3 \times 3$ min between each step except after LPR when the slides are only quickly rinsed in with PBS before nuclear counterstaining.

Abbreviations: AB, antibody; ALP, Alkaline phosphatase; DAPI, 4',6-diamidino-2-phenylindole dihydrochloride; EDTA, Ethylenediaminetetraacetic acid; LPR, liquid permanent red; PBS, phosphate buffered saline; RT, room temperature; Tris, 2-Amino-2-hydroxymethyl-propane-1,3-diol.

\section{Results}

\section{CTC-DropMount}

An overview of the CTC-DropMount technique is shown in Figure 1. Using CellSearch Profile analysis from whole blood, the recovery rate was found to be $87 \%$ on average ( $80 \%$ for 5 cells, $97 \%$ for 15 cells and $84 \%$ for 50 cells).

\section{Immunofluorescence}

Using the AlexaFluor647-labeled monoclonal CD45antibody, it was possible to separate leukocytes from CTCs under standard fluorescence microscopy. This result provides an important prerequisite for further staining and demonstrated sufficient selectivity of the method (see Figure 2). Optimal distinction between CTCs and leukocytes was achieved when combining the two filters for CK-PE and CD45-AlexaFluor647.

Criteria for ER $\alpha$-positivity defined staining of the nuclear region. The process was considered satisfactory when cells in the $\mathrm{ER}^{+}$cell line (MCF7) consistently stained positive for ER $\alpha$ with low background and marked nuclear intensity, while slides with $\mathrm{ER}^{-}{ }^{-}$cells
(SKBr3) simultaneously stained negative. Representative images are displayed in Figure 3.

HER2-staining with LPR proved highly selective (see Figure 4). The ALP-based reaction suits CTCs particularly well since endogenous enzymatic activity is negligible in these samples. Hence, the risk of false positives is insignificant. LPR permits assessment by both fluorescence and bright-field microscopy. Bright-field microscopy has the advantage of being easily accessible in most laboratories and the staining is impervious to fading. Despite the high background caused by ferrofluid remnants from CellSearch analysis, LPR staining was clearly visible. Also, if combined with CK-PE staining, bright-field microscopy was preferable due to the risk of bleed-through between PE and LPR staining in immunofluorescence analyses.

Staining of the fixed cells was optimized mainly using cells from CellSearch Profile analyses where no previous staining and permeabilization had affected the cells. However, the staining procedure was also tested following CellSearch epithelial cell analysis, and although these cells had been previously stained, the results were consistent with previously unstained cells.

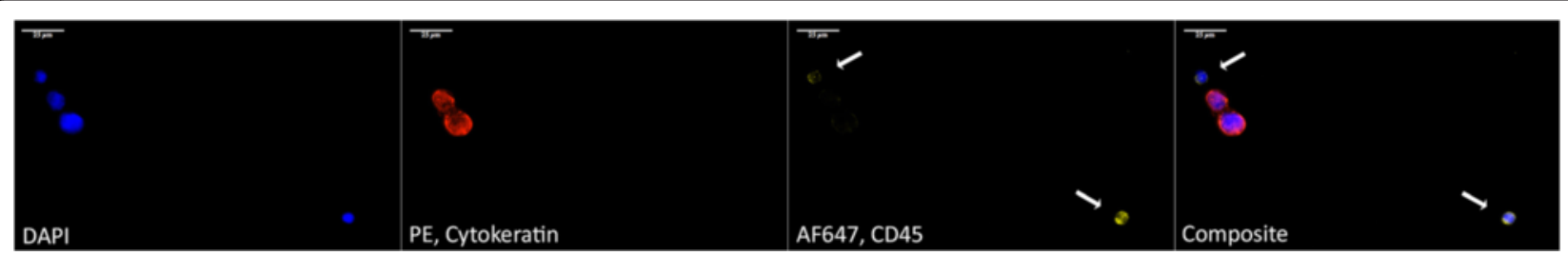

Figure 2 CD45 staining. Secondary staining of cell line cells spiked into healthy donor blood, from left to right: DAPI counterstain (fluorescent blue), cytokeratins 8, 18, and 19 (CK) stained with Phycoerythrin (red), CD45 stained with AlexaFluor 647 (yellow), and a composite of all channels. The two juxtaposed CTCs (CK-positive) stained negative for CD45, while the leukocytes (white arrows) simultaneously stained positive for CD45 and negative for CK, illustrating methodological selectivity. 


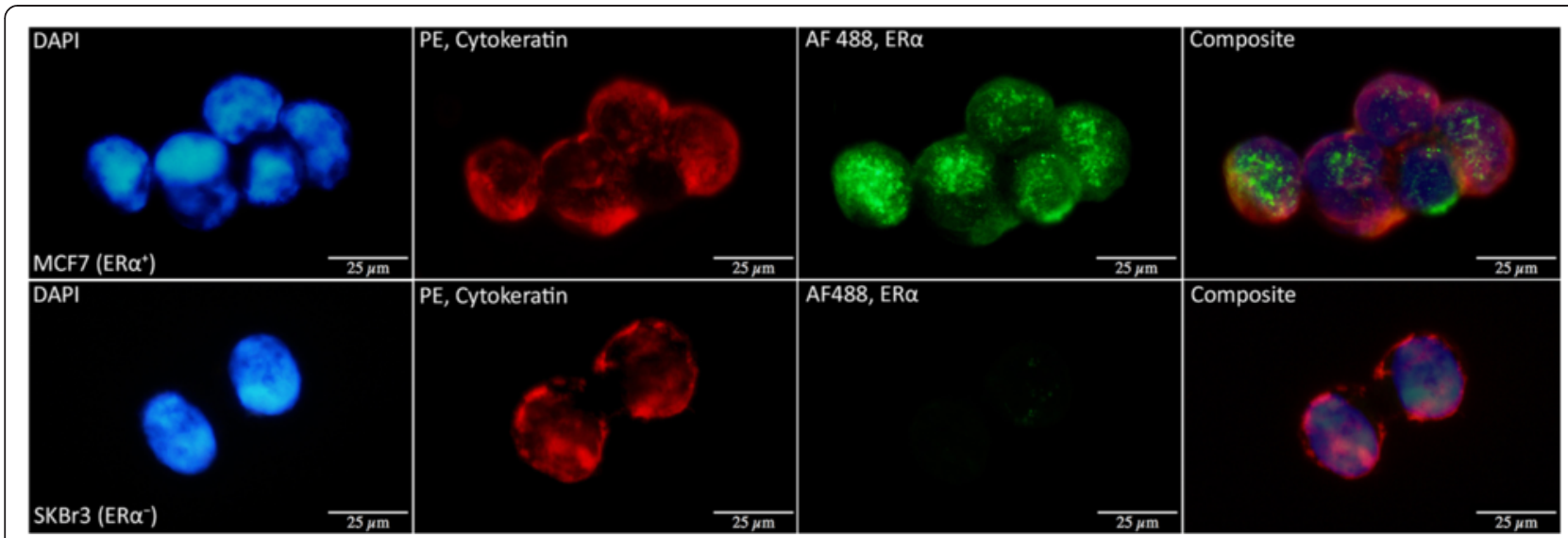

Figure 3 ERa staining of MCF7 and SKBr3 cells. Selective ERa staining demonstrated in MCF7 (ERa') and SKBr3 (ERa $)$ cells. From left to right: DAPI counterstain (fluorescent blue), cytokeratins 8, 18, and 19 (CK) stained with Phycoerythrin (red), estrogen receptor (ERa) stained with AlexaFluor 488 (green), and a composite of all channels. MCF7 showed positive nuclear staining in AlexaFluor 488 indicating positive ERa expression, while $\mathrm{SKBr} 3$ was negative.

The CTC-DropMount method could be confirmed in nine patient samples after CellSearch Profile analysis, and examples of positive ER $\alpha$ and HER2 staining can be found in Figure 5. Table 3 outlines the patients' characteristics with respect to their primary tumors, metastases, and CTC phenotypes, as well as the total number of CTCs detected by CellSearch. The majority of detected CTCs were negative for both ER $\alpha$ and HER2 expression. We observed considerable intrapatient heterogeneity in levels of biomarker expression and cell morphology, observations that are in concordance with previous research [33]. A visual comparison to corresponding CTCs in the picture galleries from CellSearch epithelial cell analyses suggested that the most intensely stained CTCs were also the most distinctly stained with the CTCDropMount method for all investigated markers.

\section{Discussion}

In this study, we present a method for secondary characterization of breast cancer CTCs after CellSearch analysis. Protocols for the clinically important predictive markers ER $\alpha$ and HER2 were optimized in breast cancer cell lines and subsequently verified in samples from patients with metastatic breast cancer. Fixation of CTCs was performed with the CTC-DropMount method described here, and ER $\alpha$ and HER2 staining protocols proved selective and consistent in our in vitro model system.

Secondary phenotypic characterization of fixed CTCs on standard microscope slides provides the possibility of concurrent morphological evaluation, assessment of the total number of cells and an estimation of the fraction of CTCs with expression of the analyzed biomarker. This gives unique information on the heterogeneity of marker expression, which is not available using PCR-based molecular methods, for example [28,35,36]. Assessment of ER $\alpha$ status in CTCs could identify patients eligible for endocrine treatment that otherwise may be overlooked (i.e. $E R \alpha^{-}$primary tumor/ER $\alpha^{+} \mathrm{CTCs}$ ). Two of the nine patients included in the in vivo validation experiments presented $\mathrm{ER \alpha}^{+}$CTCs (see Table 3, and representative images in Figure 5). Both of these samples were drawn at or just prior to initiation of treatment against metastatic disease. The phenotype of the primary tumor from one patient was classified as $\mathrm{ER}^{+}$, while the second patient had a confirmed $\mathrm{ER}^{+}$metastatic biopsy (Table 3). Conversely, detection of $\mathrm{ER}^{-}$CTCs in a patient with an $\mathrm{ER}^{+}$primary tumor might, in part, explain the lack of treatment response observed in this cohort. A similar assumption regarding HER2 gene amplification seems reasonable, since a subset of patients acquire oncogene amplification during disease progression [30]. The true number of patients suited for HER2-targeted treatment may in fact be higher than the number treated at present. This is currently being investigated in the ongoing European DETECT III and CIRCE T-DM1 studies, where the CTC HER2-positive phenotype is used as a treatment predictive marker [31]. In this study, $\mathrm{HER}^{+}{ }^{+}$CTCs were identified after 6 months of chemotherapy in a metastatic breast cancer patient with a HER2 ${ }^{-}$primary tumor and a HER2 ${ }^{-}$metastasis biopsy (see Table 3 and Figure 5).

A few previous studies have used immunological staining methods for secondary phenotypic characterization. Swennenhuis et al. fixed CTCs within the cartridge after complete CellSearch analysis using immunofluorescence and FISH analysis for successful characterization of HER2-status [37]. However, the CellTracks II analyzer had to be modified to improve the resolution and light collection. Other studies have used the FITC-channel in 


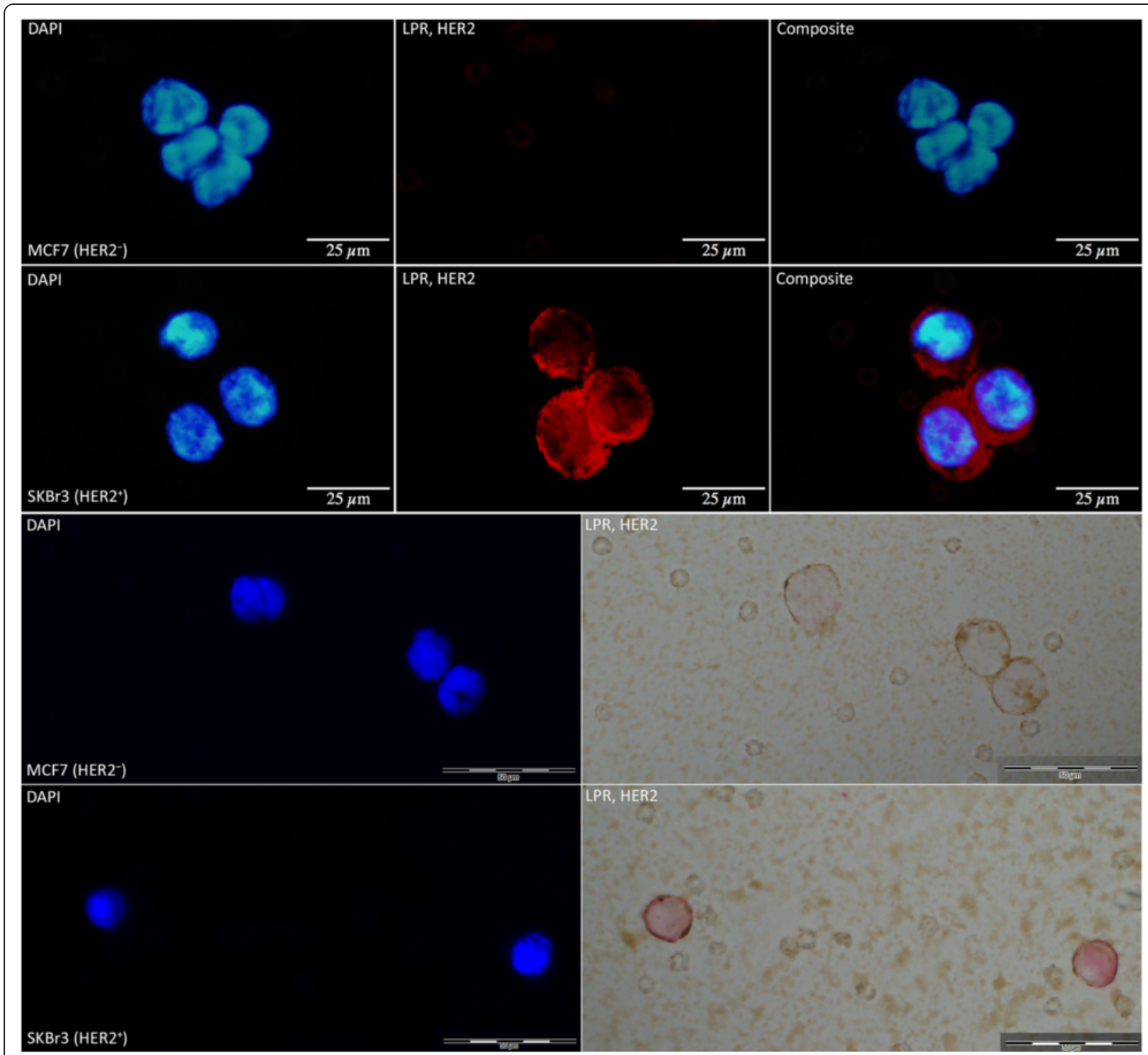

Figure 4 HER2-staining of MCF7 and SKBr3. Selective HER2 staining demonstrated in MCF7 (HER2 ${ }^{-}$) and SKBr3 (HER2 ${ }^{+}$) cells. First row: MCF7, from left to right: DAPI counterstain (fluorescent blue), HER2 stained with Liquid Permanent Red (red), and a composite of all channels. Second row: $\mathrm{SKBr} 3$, in the corresponding channels. Positive membrane staining was visible in SKBr3 cells only. Additionally, assessment of HER2 staining was also possible using bright-field microscopy, as demonstrated in the lower two rows (third row: MCF7, and fourth row: SKBr3).

the CellSearch system, where the intensity of HER2 staining is scored as negative $(0)$, very weak $(1+)$, moderate $(2+)$ or very bright $(3+)[27,38,39]$. The clinical value of specific cut-off thresholds remains to be determined. Paoletti et al. recently reported a method utilizing the CellSearch-integrated FITC-channel for analysis of ER $\alpha$, HER2, Ki67, and BCL-2 in individual blood samples with the intention of predicting resistance to endocrine therapy [40]. By implementing this approach, $7.5 \mathrm{~mL}$ of blood is required for analysis of each respective biomarker. Few studies have described methods for secondary characterization of ER $\alpha$ and HER2 in CTCs after
Ficoll density gradient separation and cytospin preparations $[17,33]$. An advantage in circumventing immunological enrichment before fixation of the cells onto microscope slides is the exemption from EpCAMdependent selection. On the other hand, the number of cells that have to be screened manually by standard microscopy is very high, thus hampering the clinical feasibility and cost effectiveness if introduced into routine clinical practice.

Fixation of CTCs on microscope slides with the described CTC-DropMount method provides the possibility to use a standard fluorescence microscope for CTC 


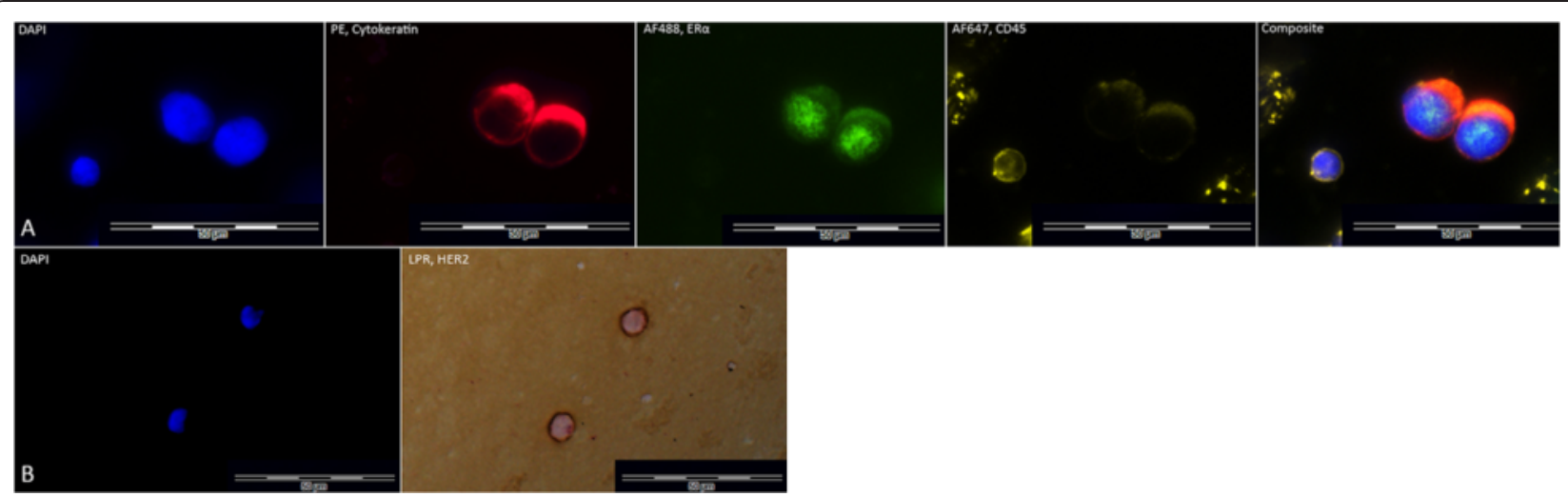

Figure $\mathbf{5}$ Immunostaining of metastatic breast cancer patient blood samples. Representative images of positive ERa and HER2 staining in clinical samples. Row A, from left to right: DAPI counterstain (fluorescent blue), cytokeratins 8, 18, and 19 (CK) stained with Phycoerythrin (red), estrogen receptor (ERa) stained with AlexaFluor 488 (green), and a composite of all channels. This patient sample (no. 4, see Table 3) was collected prior to initiation of therapy, illustrating two clustered $\mathrm{ERa}^{+} \mathrm{CTCS}$, adjacent to a solitary leukocyte located in the lower left corner. This patient was diagnosed with an $\mathrm{ERa}^{+}$metastasis. Row B, from left to right: DAPI counterstain (fluorescent blue), HER2 stained with Liquid Permanent Red (red). This patient sample (no. 1, see Table 3) was obtained following 6 months of chemotherapy, illustrating HER2 ${ }^{+}$CTCs identified by combination of fluorescence and bright-field microscopy. This patient was diagnosed with a HER2 ${ }^{-}$primary tumor and HER2 ${ }^{-}$metastasis.

characterization after immunological enrichment with the FDA-cleared CellSearch system. An advantage of the described method for secondary characterization is the scope to expand the CTC analysis to other putative predictive markers as well as to more experimental markers, for example stem cell markers, epithelial mesenchymal transition (EMT) markers or markers associated with metastasis, proliferation or apoptosis, thus increasing our knowledge of metastasis biology. Selection of single CTCs or a subset of CTCs is also possible after CTCDropMount using laser capture microdissection, for example. Subsequent single cell genomic analyses could

Table 3 Patient data for in vivo validation procedures

\begin{tabular}{|c|c|c|c|c|}
\hline $\begin{array}{l}\text { Patient } \\
\text { no. }\end{array}$ & $\begin{array}{l}\text { Primary tumor } \\
\text { phenotype }\end{array}$ & $\begin{array}{l}\text { Metastasis } \\
\text { phenotype }\end{array}$ & $\begin{array}{l}\text { Number } \\
\text { of CTCs* }\end{array}$ & $\begin{array}{l}\text { CTC } \\
\text { phenotype }\end{array}$ \\
\hline 1 & $\mathrm{ER}^{+} / \mathrm{HER}^{-}$ & $\mathrm{ER}^{-} / \mathrm{HER} 2^{-}$ & 48 & $\mathrm{ER}^{-} / \mathrm{HER}^{+}$ \\
\hline 2 & $\mathrm{ER}^{+} / \mathrm{HER}^{-}$ & $\mathrm{n} / \mathrm{a}$ & 35 & $\mathrm{ER}^{-} / \mathrm{HER}^{-}$ \\
\hline 3 & $\mathrm{ER}^{+} / \mathrm{HER} 2^{-}$ & $\mathrm{ER}^{+} / \mathrm{HER} 2^{-}$ & 3 & $n / a^{\ddagger}$ \\
\hline 4 & $\mathrm{n} / \mathrm{a}$ & $\mathrm{ER}^{+} / \mathrm{HER}^{-}$ & 12 & $\mathrm{ER}^{+} / \mathrm{HER}^{-}$ \\
\hline 5 & $\mathrm{ER}^{-} / \mathrm{HER} 2^{+}$ & $\mathrm{n} / \mathrm{a}$ & 111 & $\mathrm{ER}^{-} / \mathrm{HER} 2^{-}$ \\
\hline 6 & $\mathrm{ER}^{-} / \mathrm{HER}^{+}{ }^{+}$ & $\mathrm{n} / \mathrm{a}$ & 311 & $\mathrm{ER}^{-} / \mathrm{HER}^{-}$ \\
\hline 7 & $\mathrm{ER}^{+} / \mathrm{HER}^{-}$ & $\mathrm{ER}^{+} / \mathrm{HER}^{-}$ & 107 & $\mathrm{ER}^{+} / \mathrm{HER} 2^{-}$ \\
\hline 8 & $\mathrm{ER}^{+} / \mathrm{HER} 2^{-}$ & $\mathrm{ER}^{+} / \mathrm{HER} 2^{-}$ & 0 & Negative control \\
\hline 9 & $\mathrm{ER}^{+} / \mathrm{HER}^{-}$ & $\mathrm{ER}^{+} / \mathrm{HER} 2^{-}$ & 0 & Negative control \\
\hline
\end{tabular}

*As defined by CellSearch, single samples assessed 0-6 months from initiation of therapy against metastatic disease.

${ }^{\S}$ Phenotype according to CTC-DropMount. Criteria for biomarker positivity were $\geq 1 \mathrm{ER}^{+} \mathrm{CTC}$, and $\geq 1 \mathrm{HER}^{+} \mathrm{CTC}$.

${ }^{\ddagger}$ In this patient no CTCs were identified following secondary staining. Patients 8 , and 9 were selected as negative controls. Neither of these patients had detectable CTCs following secondary staining. open the door to an even more detailed molecular characterization of CTCs [41].

The informative advantage of heterogeneity in marker expression using secondary staining methods is also associated with the need for a prognostic cut-off value for the fraction or intensity of expression within the CTC population [17]. Using different cut-offs for marker positivity has given conflicting results regarding discordance of marker expression between primary tumors and CTCs $[17,33,42]$. Thus, the prognostic significance of marker heterogeneity in breast cancer CTCs has to be determined, and reliability on the staining methodology is of immense importance. Using the CTC-DropMount technique, we found distinct nuclear staining of ER $\alpha$ in the MCF7 cell line and used nuclear staining as a criterion for ER $\alpha$-positivity. However, to effectively determine the clinical implications, defined criteria, such as the number of CTCs to be evaluated and the fraction of $\mathrm{ER} \alpha$ - or HER2-positive CTCs, has to be decided in future clinical studies.

The cell recovery rate after CTC-DropMount fixation was $87 \%$, which is at the high end of recovery compared with studies using different methods for enrichment, fixation and detection [33,43-48]. The $80 \%$ recovery rate of five spiked cell line cells further indicates that this method could be useful in the clinical setting, where number of CTCs at the established CellSearch cut-off value ( $\geq 5 \mathrm{CTCs}$ in metastatic breast cancer) is common.

\section{Conclusions}

In conclusion, our results indicate that by retrieval of a single blood sample from patients with metastatic breast cancer it is possible to ascertain the status of important 
predictive biomarkers expressed in breast cancer CTCs. The discordance of expression between primary tumors and metastases urgently informs us that new diagnostic tools are required for optimal treatment selection in both primary and metastatic breast cancer.

\author{
Abbreviations \\ ALP: Alkaline phosphatase; APC: Allophycocyanin; CK: Cytokeratins; \\ CTCs: Circulating tumor cells; DAPI: 4',6-diamidino-2-phenylindole; \\ EDTA: Ethylenediaminetetraacetic acid; EpCAM: Epithelial cell adhesion \\ molecule; ERa: Estrogen receptor a; FBS: Fetal bovine serum; HER2: Human \\ epidermal growth factor receptor 2; LPR: Liquid permanent red; \\ PBS: Phosphate buffered saline; PE: Phycoerythrin; Tris: 2-Amino-2- \\ hydroxymethylpropane-1,3-diol.
}

\section{Competing interests}

The authors declare that they have no competing interests.

\section{Authors' contributions}

HF performed the in vitro experiments, immunofluorescent studies, figure designs and drafted the manuscript. KA conceived the study, performed the in vivo experiments and helped to draft the manuscript. LR initiated and conceived the CTC-MBC study and helped to draft the manuscript. A-MA initiated and planned the CTC-MBC study. CW initiated the conceptual design of the method. All authors read and approved the final manuscript.

\section{Acknowledgements}

We are thankful for skilled technical assistance in the CTC-lab by Kristina Lövgren and Sara Baker. Research nurse Anette Ahlin-Gullers collected blood samples from the participating patients. We are also indebted to Sofia Gruvberger-Saal for providing us with the cell lines.

This work was supported by funding from the Swedish Cancer Foundation, BioCare, the Swedish Medical Association, the Gunnar Nilsson Cancer Foundation, the Mrs Berta Kamprad Foundation, the Gyllenstierna Krapperup Foundation, and the Crafoord Foundation.

Grant numbers and sources of support

Swedish Cancer Foundation (CAN 2010/501, CAN 2010/1234, CAN 2013/533), the Gunnar Nilsson Cancer Foundation (2013/1224), the Mrs Berta Kamprad Foundation (36/2014), the Gyllenstierna Krapperup Foundation (2014/1702), the Crafoord Foundation (20100563).

\section{Author details}

'Division of Oncology and Pathology, Department of Clinical Sciences Lund, Lund University, Lund, Sweden. ${ }^{2}$ Skåne Department of Oncology, Skåne University Hospital, Lund, Sweden. ${ }^{3}$ Division of Surgery, Department of Clinical Sciences, Lund University, Lund, Sweden. ${ }^{4}$ Department of Surgery, Skåne University Hospital, Lund, Sweden.

\section{Received: 4 February 2015 Accepted: 15 April 2015}

Published online: 21 April 2015

\section{References}

1. de Bono JS, Scher HI, Montgomery RB, Parker C, Miller MC, Tissing H, et al. Circulating tumor cells predict survival benefit from treatment in metastatic castration-resistant prostate cancer. Clin Cancer Res. 2008;14(19):6302-9.

2. Cohen SJ, Punt CJA, lannotti N, Saidman BH, Sabbath KD, Gabrail NY, et al. Prognostic significance of circulating tumor cells in patients with metastatic colorectal cancer. Ann Oncol. 2009;20(7):1223-9.

3. Cristofanilli M, Budd GT, Ellis MJ, Stopeck A, Matera J, Miller MC, et al. Circulating tumor cells, disease progression, and survival in metastatic breast cancer. N Engl J Med. 2004;351(8):781-91.

4. Bidard F-C, Peeters DJ, Fehm T, Nolé F, Gisbert-Criado R, Mavroudis D, et al. Clinical validity of circulating tumour cells in patients with metastatic breast cancer: a pooled analysis of individual patient data. Lancet Oncol. 2014;15(4):406-14.

5. Goldhirsch A, Winer EP, Coates AS, Gelber RD, Piccart-Gebhart M, Thurlimann B, et al. Personalizing the treatment of women with early breast cancer: highlights of the St Gallen International Expert Consensus on the Primary Therapy of Early Breast Cancer 2013. Ann Oncol. 2013;24(9):2206-23.
6. Nadji M, Gomez-Fernandez C, Ganjei-Azar P, Morales AR. Immunohistochemistry of estrogen and progesterone receptors reconsidered: experience with 5,993 breast cancers. Am J Clin Pathol. 2005;123(1):21-7.

7. Davies C, Godwin J, Gray R, Clarke M, Cutter D, Darby S, et al. Relevance of breast cancer hormone receptors and other factors to the efficacy of adjuvant tamoxifen: patient-level meta-analysis of randomised trials. Lancet. 2011;378(9793):771-84.

8. Moy I, Lin Z, Rademaker AW, Reierstad S, Khan SA, Bulun SE. Expression of estrogen-related gene markers in breast cancer tissue predicts aromatase inhibitor responsiveness. PLoS One. 2013;8(11), e77543.

9. Osborne CK, Schiff R. Mechanisms of endocrine resistance in breast cancer. Annu Rev Med. 2011;62:233-47.

10. Lower EE, Glass EL, Bradley DA, Blau R, Heffelfinger S. Impact of metastatic estrogen receptor and progesterone receptor status on survival. Breast Cancer Res Treat. 2005;90(1):65-70.

11. Aitken SJ, Thomas JS, Langdon SP, Harrison DJ, Faratian D. Quantitative analysis of changes in ER, PR and HER2 expression in primary breast cancer and paired nodal metastases. Ann Oncol. 2010;21(6):1254-61.

12. Gomez-Fernandez C, Daneshbod Y, Nassiri M, Milikowski C, Alvarez C, Nadji M. Immunohistochemically determined estrogen receptor phenotype remains stable in recurrent and metastatic breast cancer. Am J Clin Pathol. 2008;130(6):879-82.

13. Iguchi C, Nio Y, Itakura M. Heterogeneic expression of estrogen receptor between the primary tumor and the corresponding involved lymph nodes in patients with node-positive breast cancer and its implications in patient outcome. J Surg Oncol. 2003;83(2):85-93.

14. Simmons C, Miller N, Geddie W, Gianfelice D, Oldfield M, Dranitsaris G, et al. Does confirmatory tumor biopsy alter the management of breast cancer patients with distant metastases? Ann Oncol. 2009;20(9):1499-504.

15. Fehm T, Hoffmann O, Aktas B, Becker S, Solomayer EF, Wallwiener D, et al. Detection and characterization of circulating tumor cells in blood of primary breast cancer patients by RT-PCR and comparison to status of bone marrow disseminated cells. Breast Cancer Res. 2009;11(4):R59.

16. Aktas B, Muller V, Tewes M, Zeitz J, Kasimir-Bauer S, Loehberg CR, et al. Comparison of estrogen and progesterone receptor status of circulating tumor cells and the primary tumor in metastatic breast cancer patients. Gynecol Oncol. 2011;122(2):356-60.

17. Bock C, Rack B, Kuhn C, Hofmann S, Finkenzeller C, Jager B, et al. Heterogeneity of ERalpha and ErbB2 status in cell lines and circulating tumor cells of metastatic breast cancer patients. Transl Oncol. 2012;5(6):475-85.

18. Slamon DJ, Godolphin W, Jones LA, Holt JA, Wong SG, Keith DE, et al. Studies of the HER-2/neu proto-oncogene in human breast and ovarian cancer. Science. 1989;244(4905):707-12.

19. Sjogren $S$, Inganas $M$, Lindgren A, Holmberg L, Bergh J. Prognostic and predictive value of c-erbB-2 overexpression in primary breast cancer, alone and in combination with other prognostic markers. J Clin Oncol. 1998;16(2):462-9.

20. Baselga J. Herceptin alone or in combination with chemotherapy in the treatment of HER2-positive metastatic breast cancer: pivotal trials. Oncology. 2001;61 Suppl 2:14-21.

21. Davoli A, Hocevar BA, Brown TL. Progression and treatment of HER2positive breast cancer. Cancer Chemother Pharmacol. 2010;65(4):611-23.

22. Lindstrom LS, Karlsson E, Wilking UM, Johansson U, Hartman J, Lidbrink EK, et al. Clinically used breast cancer markers such as estrogen receptor, progesterone receptor, and human epidermal growth factor receptor 2 are unstable throughout tumor progression. J Clin Oncol. 2012;30(21):2601-8.

23. Zidan J, Dashkovsky I, Stayerman C, Basher W, Cozacov C, Hadary A. Comparison of HER-2 overexpression in primary breast cancer and metastatic sites and its effect on biological targeting therapy of metastatic disease. Br J Cancer. 2005;93(5):552-6.

24. Gancberg D, Di Leo A, Cardoso F, Rouas G, Pedrocchi M, Paesmans M, et al. Comparison of HER-2 status between primary breast cancer and corresponding distant metastatic sites. Ann Oncol. 2002;13(7):1036-43.

25. Regitnig P, Schippinger W, Lindbauer M, Samonigg H, Lax SF. Change of HER-2/neu status in a subset of distant metastases from breast carcinomas. J Pathol. 2004;203(4):918-26.

26. Meng S, Tripathy D, Shete S, Ashfaq R, Saboorian H, Haley B, et al. uPAR and HER-2 gene status in individual breast cancer cells from blood and tissues. Proc Natl Acad Sci U S A. 2006;103(46):17361-5.

27. Pestrin M, Bessi S, Galardi F, Truglia M, Biggeri A, Biagioni C, et al. Correlation of HER2 status between primary tumors and corresponding 
circulating tumor cells in advanced breast cancer patients. Breast Cancer Res Treat. 2009;118(3):523-30.

28. Fehm T, Muller V, Aktas B, Janni W, Schneeweiss A, Stickeler E, et al. HER2 status of circulating tumor cells in patients with metastatic breast cancer: a prospective, multicenter trial. Breast Cancer Res Treat. 2010;124(2):403-12.

29. Liu Y, Liu Q, Wang T, Bian L, Zhang S, Hu H, et al. Circulating tumor cells in HER2-positive metastatic breast cancer patients: a valuable prognostic and predictive biomarker. BMC Cancer. 2013;13:202

30. Meng S, Tripathy D, Shete S, Ashfaq R, Haley B, Perkins S, et al. HER-2 gene amplification can be acquired as breast cancer progresses. Proc Natl Acad Sci U S A. 2004;101(25):9393-8.

31. Bidard FC, Fehm T, Ignatiadis M, Smerage JB, Alix-Panabieres C, Janni W, et al. Clinical application of circulating tumor cells in breast cancer: overview of the current interventional trials. Cancer Metastasis Rev. 2013;32(1-2):179-88.

32. Marrinucci D, Bethel K, Kolatkar A, Luttgen MS, Malchiodi M, Baehring F, et al. Fluid biopsy in patients with metastatic prostate, pancreatic and breast cancers. Phys Biol. 2012;9(1):016003

33. Babayan A, Hannemann J, Spotter J, Muller V, Pantel K, Joosse SA. Heterogeneity of estrogen receptor expression in circulating tumor cells from metastatic breast cancer patients. PLoS One. 2013;8(9), e75038.

34. Speel EJ, Schutte B, Wiegant J, Ramaekers FC, Hopman AH. A novel fluorescence detection method for in situ hybridization, based on the alkaline phosphatase-fast red reaction. J Histochem Cytochem. 1992:40(9):1299-308.

35. Van der Auwera I, Peeters D, Benoy IH, Elst HJ, Van Laere SJ, Prove A, et al. Circulating tumour cell detection: a direct comparison between the cell search system, the AdnaTest and CK-19/mammaglobin RT-PCR in patients with metastatic breast cancer. Br J Cancer. 2010;102(2):276-84.

36. Lowes LE, Allan AL. Recent advances in the molecular characterization of circulating tumor cells. Cancers (Basel). 2014;6(1):595-624.

37. Swennenhuis JF, Tibbe AGJ, Levink R, Sipkema RCJ, Terstappen LWMM. Characterization of circulating tumor cells by fluorescence in situ hybridization. Cytometry Part A. 2009;75A(6):520-7.

38. Ignatiadis M, Rothe F, Chaboteaux C, Durbecq V, Rouas G, Criscitiello C, et al. HER2-positive circulating tumor cells in breast cancer. PLoS One. 2011;6(1), e15624.

39. Riethdorf S, Muller V, Zhang L, Rau T, Loibl S, Komor M, et al. Detection and HER2 expression of circulating tumor cells: prospective monitoring in breast cancer patients treated in the neoadjuvant GeparQuattro trial. Clin Cancer Res. 2010;16(9):2634-45.

40. Paoletti C, Muniz MC, Thomas DG, Griffith KA, Kidwell KM, Tokudome N et al. Development of circulating tumor cell-endocrine therapy index in patients with hormone receptor positive breast cancer. Clin Cancer Res. 2014. Nov 7, e pub ahead of print doi: 10.1158/1078-0432.CCR-14-2781.

41. Heitzer E, Auer M, Gasch C, Pichler M, Ulz P, Hoffmann EM, et al. Complex tumor genomes inferred from single circulating tumor cells by array-CGH and next-generation sequencing. Cancer Res. 2013;7:7.

42. Punnoose EA, Atwal SK, Spoerke JM, Savage H, Pandita A, Yeh RF, et al. Molecular biomarker analyses using circulating tumor cells. PLoS One. 2010;5(9), e12517.

43. Yusa A, Toneri M, Masuda T, Ito S, Yamamoto S, Okochi M, et al. Development of a new rapid isolation device for circulating tumor cells (CTCS) using 3D palladium filter and its application for genetic analysis. PLoS One. 2014;9(2), e88821.

44. Riethdorf S, Fritsche H, Müller V, Rau T, Schindlbeck C, Rack B, et al. Detection of circulating tumor cells in peripheral blood of patients with metastatic breast cancer: a validation study of the cell search system. Clin Cancer Res. 2007;13(3):920-8.

45. Murlidhar V, Zeinali M, Grabauskiene S, Ghannad-Rezaie M, Wicha MS, Simeone DM et al. A radial flow microfluidic device for ultra-highthroughput affinity-based isolation of circulating tumor cells. Small. 2014

46. Lee A, Park J, Lim M, Sunkara V, Kim SY, Kim GH et al. All-in-one centrifugal microfluidic device for size-selective circulating tumor cell isolation with high purity. Anal Chem. 2014

47. Hillig T, Nygaard AB, Nekiunaite L, Klingelhofer J, Soletormos G. In vitro validation of an ultra-sensitive scanning fluorescence microscope for analysis of circulating tumor cells. Apmis. 2014;122(6):545-51.

48. Zhao M, Schiro PG, Kuo JS, Koehler KM, Sabath DE, Popov V, et al, An automated high-throughput counting method for screening circulating tumor cells in peripheral blood. Anal Chem. 2013;85(4):2465-71.

\section{Submit your next manuscript to BioMed Central and take full advantage of:}

- Convenient online submission

- Thorough peer review

- No space constraints or color figure charges

- Immediate publication on acceptance

- Inclusion in PubMed, CAS, Scopus and Google Scholar

- Research which is freely available for redistribution

Submit your manuscript at www.biomedcentral.com/submit 